\title{
Nonlinear PFD free of glitches and blind zone for a fast locking PLL with reduced reference spur
}

\author{
Abdul Majeed Kottampara Kuppalath ${ }^{\text {a) }}$ and Binsu J Kailath ${ }^{\text {b) }}$ \\ Indian Institute of Information Technology Design and Manufacturing (IIITDM), \\ Kancheepuram, Chennai-600127, India \\ a)edm12d001@iiitdm.ac.in \\ b)bkailath@iiitdm.ac.in
}

Abstract: A simple nonlinear PFD circuit designed to achieve reduced reference spur and faster acquisition process for a PLL is proposed in this paper. The proposed nonlinear PFD is found to offer higher gain, eliminate glitches at the output and reduce both dead zone and reset delay when compared with the operation of conventional linear PFD (CL-PFD) and conventional NL-PFD (CNL-PFD). The PLL acquisition time got reduced by $50 \%$ and $11.69 \%$ while the reference spur $45 \%$ and $38.6 \%$ with respect to the CL-PFD and CNL-PFD respectively. Reference spur of $-72.2 \mathrm{dBc}$, lock time of $1.548 \mu \mathrm{s}$, area of $0.2 \mathrm{~mm}^{2}$ and power dissipation of $6.2 \mathrm{~mW}$ are obtained for the prototype PLL using Proposed NL-PFD designed with $180 \mathrm{~nm}$ CMOS process.

Keywords: nonlinear PFD, PLL, reference spur, lock time, blind zone Classification: Integrated circuits

\section{References}

[1] M. Mansuri, et al.: IEEE J. Solid-State Circuits 37 (2002) 1331 (DOI: 10.1109/ JSSC.2002.803048).

[2] A. K. K. Majeed and B. J. Kailath: Procedia Eng. 64 (2013) 377 (DOI: 10.1016/ j.proeng.2013.09.110).

[3] J. Lan, et al.: IEEE ASICON Proc. (2009) 1117.

[4] L. C. Liu and B. H. Li: Electron. Lett. 40 (2004) 918 (DOI: 10.1049/ el:20045367).

[5] M. Lin, et al:: DTIS in Nanoscale (2007) 124.

[6] I. C. Hwang and S. G. Bae: Electron. Lett. 45 (2009) 1273 (DOI: 10.1049/ el.2009.2660).

[7] W. Rhee: IEEE ISCAS Proc. (1999) 545 (DOI: 10.1109/ISCAS.1999.780807).

[8] K. Woo, et al.: IEEE J. Solid-State Circuits 43 (2008) 379 (DOI: 10.1109/ JSSC.2007.914281).

[9] W. S. Chang, et al.: IEEE J. Solid-State Circuits 49 (2014) 2964 (DOI: 10.1109/ JSSC.2014.2359670).

[10] C. W. Hsu, et al.: IEEE Trans. Circuits Syst. I, Reg. Papers 62 (2015) 90 (DOI: 10.1109/TCSI.2014.2359719). 


\section{Introduction}

Phase locked loop (PLL) with quicker locking process and minimum reference spur is an essential component in variety of applications in modern electronics such as frequency synthesizer, synchronization of chips in GPS receiver, jitter attenuator etc. PFD is one of the important constituent blocks in the PLL system which plays a significant role in defining its lock time and reference spur. As the CL-PFD given in Fig. 1(a) cause dead zone, blind zone, lower gain, glitches at the output and associated large lock time and high reference spur [1,2], NL-PFDs have been proposed as a popular alternative $[3,4,5]$. Even though NL-PFDs offers higher gain, complicated design, complex circuits, presence of dead zone, blind zone and glitches at the output, all of these raise serious design constraints $[4,5]$. The CNLPFD given in Fig. 1(b) reported by Jinbao et al. [3] is very effective for achieving higher gain and suppressing blind zone, however dead zone and cycle slip caused by higher reset delay result in glitches at the output. Glitches would be carrying spurious tones at the output spectrum. In view of this a NL-PFD which eliminates glitches, suppresses blind zone and dead zone; and reduces reset delay is proposed in this paper.

\section{Transistor level implementation of proposed NL-PFD}

The proposed NL-PFD offers higher gain whenever phase difference $(\Delta \varphi)$ between two inputs is greater than $\pi$ and lower gain when $\Delta \varphi<\pi$. Major design constraint of eliminating glitches from the output has been addressed in the proposed circuit by using pass transistor logic and series of inverting buffers. Reset process has been accelerated by using both reset and reset bar signals simultaneously, due to which dead zone has been significantly reduced. The requirement of reset process has been eliminated, whenever phase difference is greater than $\pi$, as either UP or DN only will be at logic high so long as $\Delta \varphi$ is between $\pi$ and $2 \pi$, in the proposed NLPFD. Hence it was possible to eliminate blind zone also completely.

Transistor level implementation of proposed NL-PFD is given in Fig. 1(c). Initially, when $C L K_{\text {ref }}$ and $C L K_{v c o}$ are at logic zero, both $P_{1}$ and $P_{2}$ would be

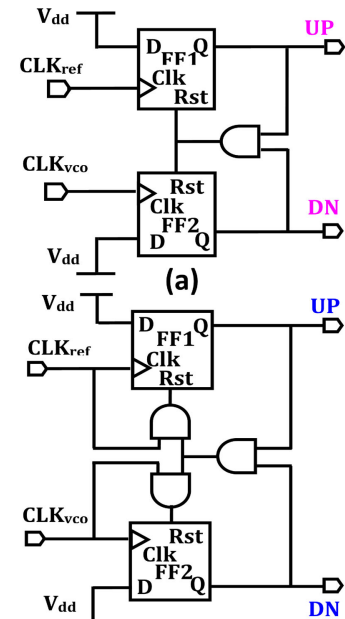

(b)

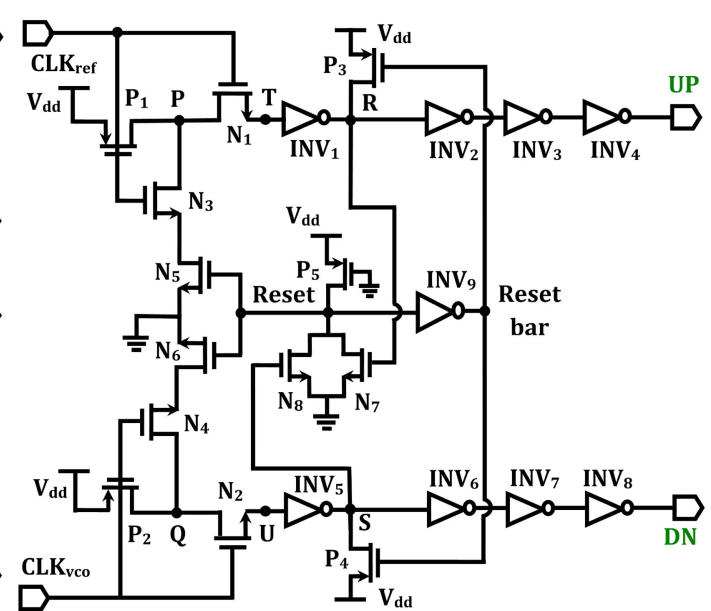

(c)

Fig. 1. (a) Block diagram of CL-PFD (b) and CNL-PFD (c) Transistor level implementation of Proposed NL-PFD. 
turned ON, pulling the nodes $\mathrm{P}$ and $\mathrm{Q}$ up to logic high. Considering the case when $|\Delta \varphi|<\pi$ and $C L K_{\text {ref }}\left(C L K_{v c o}\right)$ is leading $C L K_{v c o}\left(C L K_{\text {ref }}\right)$, at the rising edge of $C L K_{\text {ref }}\left(C L K_{v c o}\right), N_{1}$ and $N_{3}\left(N_{2}\right.$ and $\left.N_{4}\right)$ turn ON, pulling the node R (S) down to logic zero, which makes $N_{7}\left(N_{8}\right)$ turn OFF and the combination of inverters $I N V_{2}$ to $I N V_{4}\left(I N V_{6}\right.$ to $\left.I N V_{8}\right)$ causes UP (DN) signal go to logic high. UP (DN) would remain at high until the rising edge of $C L K_{v c o}\left(C L K_{\text {ref }}\right)$ wherein $N_{2}$ and $N_{4}\left(N_{1}\right.$ and $N_{3}$ ) turn $\mathrm{ON}$, pulling the node $\mathrm{S}(\mathrm{R})$ down to logic zero, and making $N_{8}\left(N_{7}\right)$ turn OFF. When $N_{7}$ and $N_{8}$ turn OFF, reset signal would be pulled up to logic high and reset-bar signal down to logic zero. Simultaneously the nodes $\mathrm{R}$ and $\mathrm{S}$ would be pulled up to logic high making both UP and DN to become logic zero. This prevents the condition of both UP and DN simultaneously reaching logic high. And the reset signal ensures that both the nodes $\mathrm{P}$ and $\mathrm{Q}$ would be pulled down to logic zero through $N_{3}-N_{5}$ and $N_{4}-N_{6}$ respectively.

Considering the case when $|\Delta \varphi|>\pi$ and $C L K_{\text {ref }}\left(C L K_{v c o}\right)$ is leading $C L K_{v c o}$ $\left(C L K_{\text {ref }}\right)$, at the rising edge of $C L K_{\text {ref }}\left(C L K_{v c o}\right)$, node R (S) would be at logic low, $N_{7}\left(N_{8}\right)$ would be OFF and UP (DN) would be at logic high. Even when $C L K_{\text {ref }}$ $\left(C L K_{v c o}\right)$ makes a high to low transition which happens before $C L K_{v c o}\left(C L K_{r e f}\right)$ makes the low to high transition, UP (DN) would remain at logic high as $N_{1}\left(N_{2}\right)$ turns OFF. At the rising edge of $C L K_{v c o}\left(C L K_{\text {ref }}\right)$, node $\mathrm{S}(\mathrm{R})$ would be at logic zero and $N_{8}\left(N_{7}\right)$ would be OFF. Under the condition when both $N_{7}$ and $N_{8}$ are simultaneously OFF, even though reset signal would be at logic high and reset-bar signal at logic zero, only DN (UP) signal would get reset to logic zero while UP (DN) would remain at logic high as $N_{1}\left(N_{2}\right)$ is OFF, and this status viz UP $=1$ and $\mathrm{DN}=0(\mathrm{UP}=0$ and $\mathrm{DN}=1)$ would continue until the phase difference between $C L K_{\text {ref }}\left(C L K_{v c o}\right)$ and $C L K_{v c o}\left(C L K_{\text {ref }}\right)$ becomes less than $\pi$. Switching characteristics of the circuit is discussed in the following section.

\section{Simulation results of proposed NL-PFD, CL-PFD and CNL-PFD}

The transient analysis of CL-PFD, CNL-PFD and proposed NL-PFD are presented in Fig. 2(a). It has been found that the magnitude of signals at nodes $\mathrm{T}$ and $\mathrm{U}$ marked in circuit in Fig. 1(c) have had a peak of $1.2 \mathrm{~V}$ and not $1.8 \mathrm{~V}\left(V_{d d}\right)$, due to the $V_{T N}$ drop across the NMOS pass transistor $\left(N_{1}, N_{2}\right)$, while the amplitude of glitches at same nodes $\mathrm{T}$ and $\mathrm{U}$ is has been found to be about $0.6 \mathrm{~V}$. Output voltage

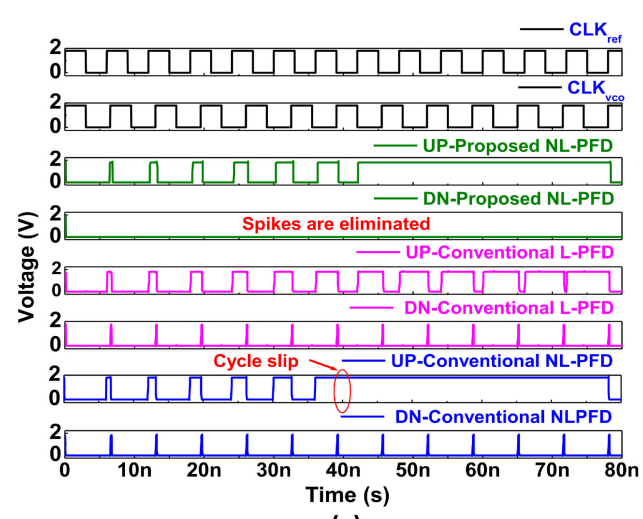

(a)

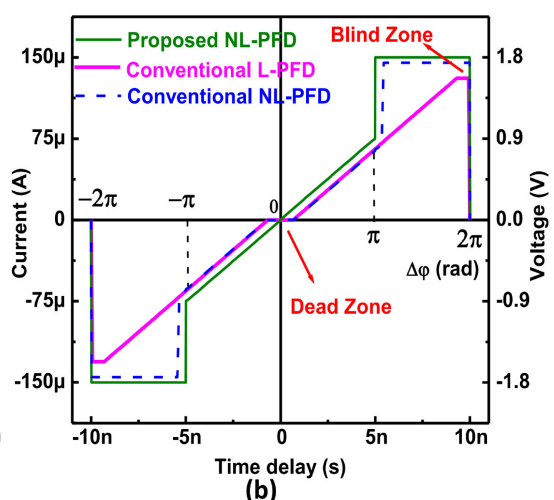

(b)

Fig. 2. Proposed NL-PFD, CL-PFD and CNL-PFD (a) Transient response (b) Transfer characteristics. 


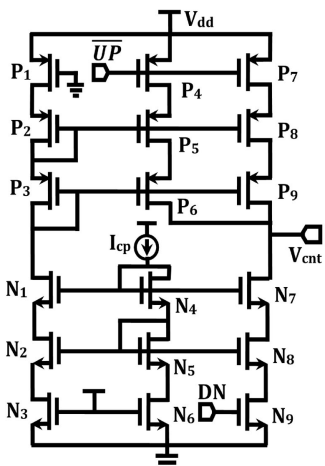

(a)

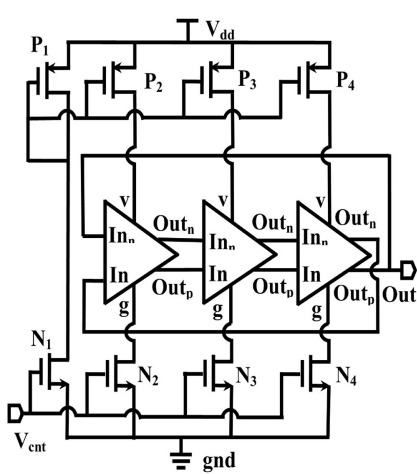

(b)

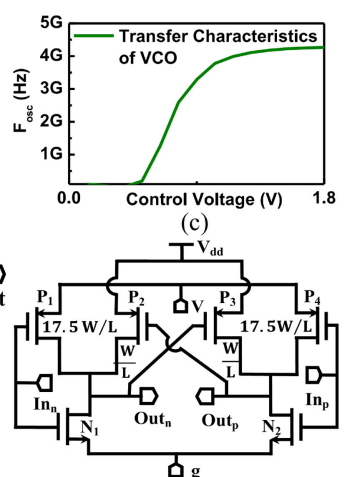

(d)

Fig. 3. Transistor level implementation of (a) CP (b) VCO (d) Delay cell (c) Transfer Characteristics of VCO.

is restored to $V_{d d}$ by adding a buffer with four inverters $I N V_{1}$ through $I N V_{4}$ for UP and $I N V_{5}$ through $I N V_{8}$ for DN respectively. The buffers are also found to be effective in eliminating glitches at the output of UP and DN as well. Another factor which is effective in eliminating glitches is the reduction in reset delay obtained due to removal of delay element as well as operation of reset and reset-bar signals simultaneously. This ensures fast reset process as neither UP nor DN get enough time to reach logic high simultaneously.

It can be observed that for an initial duration of $36 \mathrm{~ns}$, when the phase difference between $C L K_{\text {ref }}$ and $C L K_{v c o}$ is less than $\pi$, the pulse width of UP signal is increasing as the phase difference $\Delta \varphi$ is increasing. After $36 \mathrm{~ns}$, when the phase difference is greater than $\pi$ with $C L K_{\text {ref }}$ leading $C L K_{v c o}$, the UP signal is held at high until the phase difference become less than $\pi$ which happens after about five cycles of $C L K_{r e f}$ and $C L K_{v c o}$. Similar characteristics have been obtained for DN signal when $C L K_{v c o}$ is leading $C L K_{\text {ref }}$. The major achievement in the proposed design is that, glitches are completely eliminated at the output, whereas it does exist for both CL-PFD and CNL-PFD.

The output voltage as a function of input phase difference for proposed NLPFD is presented in Fig. 2(b) and can be expressed as:

$$
f(\varphi)= \begin{cases}\frac{V_{d d}}{2 \pi} \varphi & -\pi<\varphi<\pi \\ V_{d d} & |\pi|<\varphi<|2 \pi|\end{cases}
$$

The function $f(\varphi)$ representing both linear and nonlinear operating regions is determined using Fourier series as:

$$
f(\varphi)=\sum_{0}^{\infty} \frac{V_{d d}}{2 \pi}\left[\cos \left(\frac{n \pi}{2}\right)-2 \cos (n \pi)+\frac{2}{n \pi} \sin \left(\frac{n \pi}{2}\right)\right] \sin \left(\frac{n \varphi}{2}\right) \quad-2 \pi<\varphi<2 \pi
$$

Equation (2) has been plotted in MATLAB as $\varphi$ is varied from $-2 \pi$ to $2 \pi$, with $n$ varying from 1 to 200 and is plotted in Fig. 2(b). $\varphi$-V characteristics of proposed NL-PFD, CL-PFD and CNL-PFDs have also been determined from circuit simulation by introducing delay between $C L K_{v c o}$ and $C L K_{\text {ref }}$ and is also appended in Fig. 2(b) with time delay varying from $-10 \mathrm{~ns}$ to $10 \mathrm{~ns}$ corresponding to phase difference varying from $-2 \pi$ to $2 \pi$. It can be observed that the results from both 


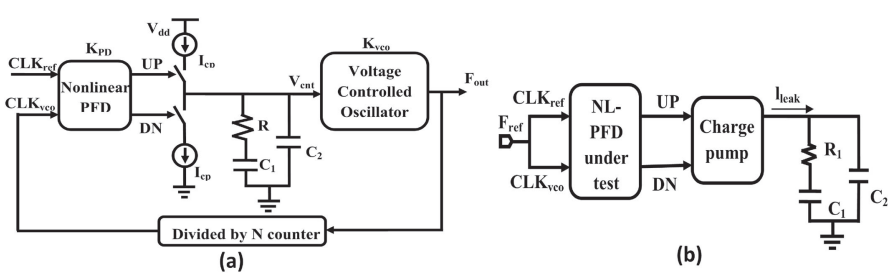

Fig. 4. (a) PLL Diagram (b) Leakage current simulation setup

Cadence and MATLAB simulations are exactly matching with each other. It can also be observed that dead zone and blind zone are present in CL-PFD while only dead zone is present in CNL-PFD. More importantly both dead zone and blind zone get completely eliminated from the $\varphi-\mathrm{V}$ characteristics of the proposed NL-PFD and this result in increased gain.

\section{PLL circuit simulation using proposed NL-PFD:}

In order to compare the performance of PLL built with proposed NL-PFD, CL-PFD and CNL-PFD, three PLLs of similar structure as given in Fig. 4(a) has been implemented using $180 \mathrm{~nm}$ CMOS technology in Cadence. Each of proposed NLPFD, CL-PFD and CNL-PFD is used as the PFD in each while a modified cascode configuration given in Fig. 3(a) is used as the charge pump. Second order loop filter has been implemented with $R_{1}=1 \mathrm{~K} \Omega, C_{1}=277 \mathrm{pF}$ and $C_{2}=25 \mathrm{pF}$. Current starved Voltage Controlled ring Oscillator given in Fig. 3(b), has been implemented using 3 stage CMOS differential delay cell given in Fig. 3(d). Transfer characteristics of VCO is plotted in Fig. 3(c), from which the gain of VCO has been computed to be $K_{v c o}=20 \mathrm{Grad} V^{-1}$. PLL output has been scaled down to reference frequency of $20 \mathrm{MHz}$ using divide by 128 counter. Post layout simulation have been performed and the lock time has been calculated to be $1.548 \mu \mathrm{s}, 3.1 \mu \mathrm{s}$ and $1.753 \mu \mathrm{s}$ for the PLLs using proposed NL-PFD, CL-PFD and CNL-PFD respectively and the graphs are plotted in Fig. 6(b). Final placement of the PLL using proposed NL-PFD is found to require a chip area of $0.2 \mathrm{~mm}^{2}$ with $485 \mu \mathrm{m}$ in width and $414 \mu \mathrm{m}$ in length as given in Fig. 6(a) while its total power dissipation from the chip is found to be $6.2 \mathrm{~mW}$.

Output spectrum of PLLs have been plotted to determine the reference spur in each case and is found to be $-72.2,-39.52$ and $-44.3 \mathrm{dBc} @ 20 \mathrm{MHz}$ offset from the PLL output frequency of $2.56 \mathrm{GHz}$ for the PLL using proposed NL-PFD, CLPFD and CNL-PFD respectively. The spectra for CL-PFD and CNL-PFD are given

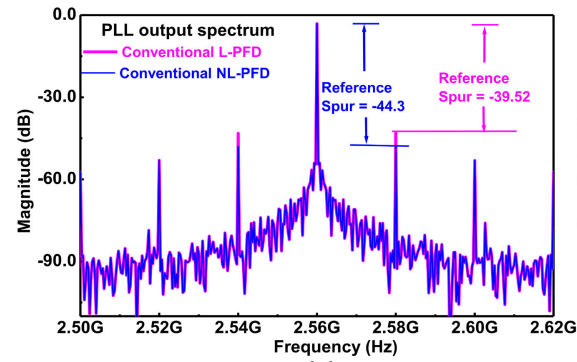

(a)

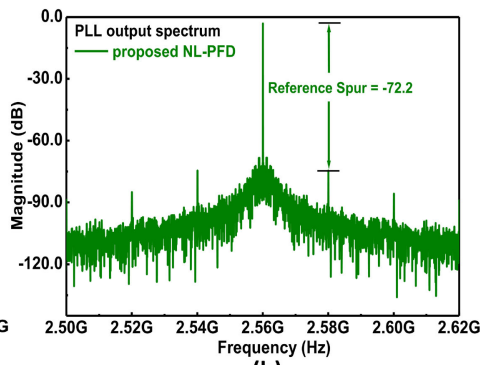

(b)

Fig. 5. Output spectrum of PLL using (a) CL-PFD and CNL-PFD (b) proposed NL-PFD 


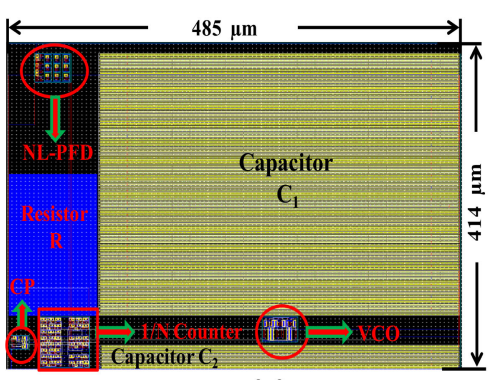

(a)

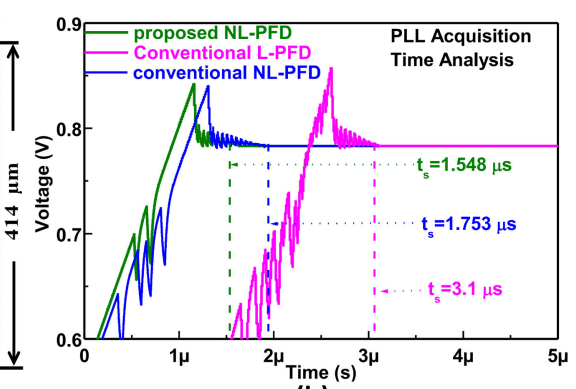

(b)

Fig. 6. (a) PLL Layout (b) Acquisition process of PLL using proposed NL-PFD, CL-PFD and CNL-PFD.

in Fig. 5(a) with that of proposed one in Fig. 5(b). Reference spur also has been determined analytically from leakage current. Schematic of the setup for simulation of leakage current of the PFD under test is given in Fig. 4(b) [6] and it is calculated as $5.4 \mathrm{nA}$ for proposed NL-PFD. Hence the phase offset could be determined as [7]:

$$
\phi_{e}=2 \pi \times \frac{I_{\text {leak }}}{I_{C P}}
$$

And the reference spur could be expressed as [7];

$$
P_{r}=20 \log \left[\frac{N \phi_{e} f_{L B W}}{f_{r e f} \sqrt{2}}\right]-20 \log \left[\frac{f_{r e f}}{f_{p l}}\right]
$$

The reference spur for the PLL using proposed NL-PFD is calculated to be $-72.08 \mathrm{dBc}$ and is found to be exactly matching with the data from circuit simulation as presented in Fig. 5(b). Results obtained from proposed circuit, CLPFD and CNL-PFD and reported works are compared in Table I and the proposed circuit is found to achieve better performance parameters when compared with the conventional and reported data.

Table I. Performance comparison of PLL

\begin{tabular}{llcllll}
\hline Parameter & $\begin{array}{c}\text { Con. } \\
\text { L-PFD }\end{array}$ & $\begin{array}{c}\text { Con. } \\
\text { NL-PFD }\end{array}$ & {$[10]$} & {$[8]$} & {$[9]$} & $\begin{array}{c}\text { This } \\
\text { work }\end{array}$ \\
\hline Lock Time $(\mu \mathrm{s})$ & 3.1 & 1.753 & 4 & 20 & $21 \mathrm{~K}$ & 1.548 \\
\hline Reference Spur $(\mathrm{dBc})$ & -39.52 & -44.3 & -41 & -54 & -55 & -72.2 \\
\hline
\end{tabular}

\section{Conclusion}

A simple NL-PFD circuit suitable for fast locking and reduced reference spur PLL has been proposed in this paper. The proposed NL-PFD is found to offer higher gain, eliminate glitches at the output and reduce both dead zone and reset delay when compared with the operation of CL-PFD and CNL-PFD. The PLL acquisition time for proposed circuit got reduced by 50\% with respect to CL-PFD, and $11.69 \%$ to CNL-PFD while the reference spur by $45 \%$ and $38.6 \%$ respectively. Reference spur of $-72.2 \mathrm{dBc}$, lock time of $1.548 \mu \mathrm{s}$, area of $0.2 \mathrm{~mm}^{2}$ and power dissipation of $6.2 \mathrm{~mW}$ are obtained for the prototype PLL using Proposed NL-PFD designed with $180 \mathrm{~nm}$ CMOS process. 